Bull. Korean Math. Soc. 49 (2012), No. 5, pp. 911-921

http://dx.doi.org/10.4134/BKMS.2012.49.5.911

\title{
RADIAL OSCILLATION OF LINEAR DIFFERENTIAL EQUATION
}

\author{
ZhaOJUn Wu
}

\begin{abstract}
In this paper, the radial oscillation of the solutions of higher order homogeneous linear differential equation

$$
f^{(k)}+A_{n-2}(z) f^{(k-2)}+\cdots+A_{1}(z) f^{\prime}+A_{0}(z) f=0
$$

with transcendental entire function coefficients is studied. Results are obtained to extend some results in [Z. Wu and D. Sun, Angular distribution of solutions of higher order linear differential equations, J. Korean Math. Soc. 44 (2007), no. 6, 1329-1338].
\end{abstract}

\section{Introduction and main results}

In this paper, the meromorphic function always means a function being meromorphic in the whole complex plane $\mathbb{C}$. Assume that the basic definitions, theorems and standard notations of the Nevanlinna theory for meromorphic function (see [11], [22] or [24]) are known. There have appeared many papers on the global theory of complex differential equations which were studied from the point of view of Nevanlinna theory, since 1982 when the article by Bank and Laine [1] appeared in Trans. Amer. Math. Soc. We refer the reader to the books by Laine [12], and by Gao etc. [6]. The first general research on the radial oscillation theory of the solutions of

$$
f^{\prime \prime}-A(z) f=0
$$

is due to Wang [17] and $\mathrm{Wu}$ [19] respectively. Here, we recall some definitions by Wang [17] as follows (also see Rossi and Wang [14]).

Definition 1 (Sectorial exponent of convergence). For $a \in \mathbb{C}_{\infty}:=\mathbb{C} \cup\{\infty\}$, define

$$
\lambda_{\alpha, \beta}(f, a)=\limsup _{r \rightarrow \infty} \frac{\log n(r, \Omega(\alpha, \beta), f=a)}{\log r},
$$

Received April 29, 2011; Revised September 27, 2011.

2010 Mathematics Subject Classification. Primary 30D30; Secondary 30D35, 34M20.

Key words and phrases. radial exponent of convergence, iterated order, sectorial oscillation, Borel direction.

(C)2012 The Korean Mathematical Society 
where $\Omega(\alpha, \beta)=\{z \mid \alpha<\arg z<\beta\}, 0<\beta-\alpha \leq \pi$ and $n(r, \Omega(\alpha, \beta), f=a)$ is the number of the roots of $f(z)-a=0$ in $\Omega(\alpha, \beta) \cap\{|z|<r\}$, counting multiplicities. Especially, when $a=0$, we write $\lambda_{\alpha, \beta}(f)=\lambda_{\alpha, \beta}(f, 0)$.

Definition 2 (Radial exponent of convergence). For any $\theta \in[0,2 \pi)$ and $a \in$ $\mathbb{C}_{\infty}$, we define

$$
\lambda_{\theta}(f, a)=\lim _{\varepsilon \rightarrow 0^{+}} \lambda_{\theta-\varepsilon, \theta+\varepsilon}(a, f) .
$$

Especially, when $a=0$, we write $\lambda_{\theta}(f)=\lambda_{\theta}(f, 0)$.

In 1994, Wu [19] had proved the following theorem.

Theorem A ([19]). Let $A(z)$ be a transcendental entire function of finite order in the plane and let $f_{1}, f_{2}$ be two linearly independent solutions of (1). Set $E=$ $f_{1} f_{2}$. Then $\lambda_{\theta}(E)=+\infty$, if and only if $\lim \sup _{r \rightarrow \infty} \frac{\log \log M\left(r, \Omega_{\theta, \varepsilon}, E\right)}{\log r}=+\infty$ for any $\varepsilon>0$, where $M\left(r, \Omega_{\theta, \varepsilon}, E\right)=\sup \left\{\left|E\left(t e^{i \tau}\right)\right|: \theta-\varepsilon \leq \tau \leq \theta+\varepsilon, 1 \leq t \leq r\right\}$.

Recently, $\mathrm{Wu}[18]$ proved the following theorem on connection of the radial exponent of convergence of zeros with Borel direction of the product of a solution base of (1).

Theorem B ([18]). Let $A(z)$ be a transcendental entire function of finite order in the plane and $f_{1}, f_{2}$ be two linearly independent solutions for (1). Let $E=$ $f_{1} f_{2}$. Suppose that the exponent of convergence of zero-sequence $\lambda(E)$ is $\infty$. Then $L: \arg z=\theta_{0}$ is an infinity order Borel direction of $E$ if and only if $\lambda_{\theta_{0}}(E)=\infty$.

For $k \geq 2$, we consider the homogeneous linear differential equation

$$
f^{(k)}+A_{k-2} f^{(k-2)}+\cdots+A_{0} f=0,
$$

where $A_{0}, \ldots, A_{k-2}$ are entire functions with $A_{0} \not \equiv 0$. Bernal [3] studied the iterated $p$-order of solutions of (2). In this paper, we shall study the connection of the radial exponent of convergence of zeros with Borel direction of the product of a solution base of linear differential equation (2) with entire coefficients of finite iterated $p$-order. For the sake of convenience, we define inductively (see [3], [10], [11], [15]), for $r \in[0,+\infty)$, $\exp ^{[1]} r=e^{r}$ and $\exp ^{[n+1]} r=\exp \left(\exp ^{[n]} r\right), n \in \mathbb{N}$. For all $r$ sufficiently large, we define $\log ^{[1]} r=\log r$ and $\log ^{[n+1]} r=\log \left(\log ^{[n]} r\right), n \in \mathbb{N}$. We also denote $\exp ^{[0]} r=r=\log ^{[0]} r, \log ^{[-1]} r=\exp ^{[1]} r$ and $\exp ^{[-1]} r=\log ^{[1]} r$. We recall the following definitions and remarks.

Definition $3([11,15])$. The iterated $p$-order $\sigma_{p}(f)$ of a meromorphic function $f(z)$ is defined by

$$
\sigma_{p}(f)=\limsup _{r \rightarrow \infty} \frac{\log ^{[p]} T(r, f)}{\log r} \quad(p \in \mathbb{N}) .
$$


Remark 1 ([4]). (1) If $p=1$, then we denote $\sigma_{1}(f)=\sigma(f) ;(2)$ If $p=2$, then we denote the so-called hyper order by $\sigma_{2}(f)$; (3) If $f(z)$ is an entire function, then

$$
\sigma_{p}(f)=\limsup _{r \rightarrow \infty} \frac{\log ^{[p+1]} M(r, f)}{\log r} .
$$

Definition $4([3,15])$. The growth index of the iterated order of a meromorphic function $f(z)$ is defined by

$$
i(f)= \begin{cases}0 & \text { if } f \text { is rational, } \\ \min \left\{n \in \mathbb{N}: \sigma_{n}(f)<\infty\right\} & \text { if } f \text { is transcendental and } \\ & \sigma_{n}(f)<\infty \text { for some } n \in \mathbb{N} \\ \infty & \text { if } \sigma_{n}(f)=\infty \text { for all } n \in \mathbb{N} .\end{cases}
$$

In [3], Bernal prove the following theorem.

Theorem C ([3]). Assume that $A_{0}, \ldots, A_{n-1}$ are entire functions and $p=$ $\max \left\{i\left(A_{j}\right), j=0, \ldots, k-1\right\}$. Set

$$
L(f):=f^{(n)}+A_{n-1} f^{(n-1)}+A_{n-2}(z) f^{(n-2)}+\cdots+A_{1}(z) f^{\prime}+A_{0}(z) f .
$$

If $p>0$, let $\sigma=\max \left\{\sigma_{p}\left(A_{j}\right), j=0, \ldots, k-1\right\}$, then

(i) $\delta \leq 1+p$, where $\delta=\sup \{i(f): L(f)=0\}$.

(ii) if $p<\infty$, then $\gamma_{p+1} \leq \sigma$, where $\gamma_{p+1}=\sup \left\{\sigma_{p+1}(f): L(f)=0\right\}$.

Definition 5 ([11, 15]). The iterated convergence exponent of the sequence of $a$-points $\left(a \in \mathbb{C}_{\infty}\right)$ is defined by

$$
\lambda_{n}(f-a)=\lambda_{n}(f, a)=\limsup _{r \rightarrow \infty} \frac{\log ^{[n]} N\left(r, \frac{1}{f-a}\right)}{\log r} \quad(n \in \mathbb{N}) .
$$

Remark $2([4]) . \quad \lambda_{1}(f-a)=\lambda(f-a) ; \lambda_{1}(f, 0)=\lambda(f) ; \lambda_{p}(f, 0)=\lambda_{p}(f)$.

Definition 6. The sectorial iterated convergence exponent of the sequence of $a$-points $\left(a \in \mathbb{C}_{\infty}\right)$ is defined by

$$
\lambda_{n, \alpha, \beta}(f-a)=\lambda_{n, \alpha, \beta}(f, a)=\limsup _{r \rightarrow \infty} \frac{\log ^{[n]} n(r, \Omega(\alpha, \beta), f=a)}{\log r} \quad(n \in \mathbb{N}) .
$$

The radial iterated convergence exponent of the sequence of $a$-points $\left(a \in \mathbb{C}_{\infty}\right)$ is defined by

$$
\lambda_{n, \theta}(f-a)=\lambda_{n, \theta}(f, a)=\lim _{\varepsilon \rightarrow 0^{+}} \lambda_{n, \theta-\varepsilon, \theta+\varepsilon}(f, a) \quad(n \in \mathbb{N}) .
$$

Remark 3. $\lambda_{1, \alpha, \beta}(f-a)=\lambda_{\alpha, \beta}(f-a) ; \lambda_{1, \theta}(f-a)=\lambda_{\theta}(f-a)$.

Definition 7. Let $p \in \mathbb{N}$, and $f(z)$ be a meromorphic function of iterated $p$ order $\rho(0<\rho \leq \infty)$. A ray $L: \arg z=\theta$ is called a Borel direction of iterated 
$p$-order $\rho$ of $f$, if no matter how small the positive number $0<\varepsilon<\pi / 2$ is, for each value $a \in \mathbb{C}_{\infty}$, holds

$$
\limsup _{r \rightarrow \infty} \frac{\log ^{[p]} n(r, \Omega(\theta-\varepsilon, \theta+\varepsilon), f=a)}{\log r}=\rho,
$$

with at most two exceptional values $a$.

Remark 4. When $p=1$, Borel direction of iterated $p$-order $\rho$ of $f$ is called a Borel direction of order $\rho$ of $f$. When $p=2$, Borel direction of iterated $p$-order $\rho$ of $f$ is called a Borel direction of hyper order $\rho$ of $f$.

In [23], the author prove the following theorem.

Theorem D ([23]). Assume that some (or all) of $A_{0}, \ldots, A_{k-2}$ are transcendental entire functions of finite order growth and equation (2) possesses a solution base $f_{1}, f_{2}, \ldots, f_{k}$. Set $E=f_{1} \cdots f_{k}$. Then $\lambda_{\theta}(E)=+\infty$, if and only if

for any $\varepsilon>0$.

$$
\limsup _{r \rightarrow \infty} \frac{\log \log M\left(r, \Omega_{\theta, \varepsilon}, E\right)}{\log r}=+\infty
$$

This result and Theorem B motivate the present author to prove the following theorem in [20].

Theorem E. Assume that some (or all) of $A_{0}, \ldots, A_{k-2}$ are transcendental entire functions of finite order growth and equation (2) possesses a solution base $f_{1}, f_{2}, \ldots, f_{k}$. Set $E=f_{1} \cdots f_{k}$. Suppose that the exponent of convergence of zero-sequence $\lambda(E)=\infty$ and $E$ is an entire function of hyper order $\rho(0<$ $\rho<\infty)$. Then $\lambda_{2, \theta}(E)=\rho$ if and only if $L: \arg z=\theta$ is a Borel direction of hyper order $\rho$ of $E$.

In this paper, we shall continue to research the radial oscillation of the solutions of (2). In fact, we shall prove the following theorem.

Theorem 1. Assume that some (or all) of $A_{0}, \ldots, A_{k-2}$ are transcendental entire functions, and $p=\max \left\{i\left(A_{j}\right), j=0,1, \ldots, k-2\right\}<\infty$. If equation (2) possesses a solution base $f_{1}, f_{2}, \ldots, f_{k}$ and set $E=f_{1} \cdots f_{k}$. Then $i(E) \leq$ $(p+1)$. If $\sigma_{(p+1)}(E)=\rho>0$, then the following statements are equivalent:

(i) $L: \arg z=\theta$ is a Borel direction of iterated $(p+1)$-order $\rho$ of $E$;

(ii) $\lambda_{(p+1), \theta}(E)=\rho$;

(iii) $\limsup _{r \rightarrow \infty} \frac{\log ^{[p+2]} M\left(r, \Omega_{\theta, \varepsilon}, E\right)}{\log r}=\rho$ for any $\varepsilon>0$.

By Theorem $\mathrm{C}$, we know that $i(E) \leq p+1$. When $k=2$ and $p=1$, the equivalence of (i) and (ii) is a precise version of Theorem B in the case of $\sigma_{2}(E)=\rho>0$. When $k \geq 2$ and $p=1$, the equivalence of (i) and (ii) is Theorem E. When $k=2$ and $p=1$, the equivalence of (ii) and (iii) has been obtained by Huang and Chen [7] in an weakly form. When $k \geq 2$ and $p=1$, 
the equivalence of (ii) and (iii) has been obtained by Huang and Chen [8] in an weakly form. Most recently, Zheng [24] give a system research about the equivalence of (i) and (ii) by using the proximate order of Qinglai Hiong (see [5]). Here, the innovative point is the equivalence of (ii) and (iii).

\section{Some lemmas}

The proof of Theorem 1 requires the Nevanlinna theory in an angular domain. Let $f(z)$ be a meromorphic function and $\bar{\Omega}(\alpha, \beta)=\{z \mid \alpha \leq \arg z \leq \beta\}$ be an angular domain, where $0<\beta-\alpha \leq 2 \pi$. Nevanlinna defined the following notations (see e.g. [5], [13], [24]).

$$
\begin{gathered}
A_{\alpha, \beta}(r, f)=\frac{k}{\pi} \int_{1}^{r}\left(\frac{1}{t^{k}}-\frac{t^{k}}{r^{2 k}}\right)\left\{\log ^{+}\left|f\left(t e^{i \alpha}\right)\right|+\log ^{+}\left|f\left(t e^{i \beta}\right)\right|\right\} \frac{d t}{t} \\
B_{\alpha, \beta}(r, f)=\frac{2 k}{\pi r^{k}} \int_{\alpha}^{\beta} \log ^{+}\left|f\left(r e^{i \theta}\right)\right| \sin k(\theta-\alpha) d \theta \\
C_{\alpha, \beta}(r, f)=2 \sum_{b_{v} \in \triangle}\left(\frac{1}{\left|b_{v}\right|^{k}}-\frac{\left|b_{v}\right|^{k}}{r^{2 k}}\right) \sin k\left(\beta_{v}-\alpha\right),
\end{gathered}
$$

where $k=\frac{\pi}{\beta-\alpha}, 1 \leq r<\infty$ and $b_{v}=\left|b_{v}\right| e^{i \theta_{v}}$ are the poles of $f(z)$ in the sector $\triangle:=\{z: 1<|z|<r, \alpha<\arg z<\beta\}$, each pole $b_{v}$ occurs in the sum $\sum_{b_{v} \in \triangle}$ as many times as it's order. When pole $b_{v}$ occurs in the sum $\sum_{b_{v} \in \Delta}$ only once, we denote it by $\bar{C}_{\alpha, \beta}(r, f)$. Moreover, for $r>1$, we define

$$
D_{\alpha, \beta}(r, f)=A_{\alpha, \beta}(r, f)+B_{\alpha, \beta}(r, f), \quad S_{\alpha, \beta}(r, f)=C_{\alpha, \beta}(r, f)+D_{\alpha, \beta}(r, f) .
$$

For the sake of simplicity, we omit the subscript of all the notations and use $A(r, f), B(r, f), C(r, f), D(r, f)$ and $S(r, f)$ to replace $A_{\alpha, \beta}(r, f), B_{\alpha, \beta}(r, f)$, $C_{\alpha, \beta}(r, f), D_{\alpha, \beta}(r, f)$ and $S_{\alpha, \beta}(r, f)$ respectively. In the following, some properties of $S(r, f)$ are given.

Lemma 1 ([24]). Let $f(z)$ be a nonconstant meromorphic function and $\bar{\Omega}(\alpha, \beta)$ be an angular domain, where $0<\beta-\alpha \leq 2 \pi$. Then for any value $a \in \mathbb{C}$, we have

$$
S\left(r, \frac{1}{f-a}\right)=S(r, f)+\varepsilon(r, a),
$$

where $\varepsilon(r, a)=O(1)$ as $r \rightarrow \infty$.

Lemma 2 ([24]). Let $f(z)$ be a meromorphic function and $\bar{\Omega}(\alpha, \beta)$ be an angular domain, where $0<\beta-\alpha \leq 2 \pi$. Then for arbitrary $q$ distinct $a_{j} \in \mathbb{C}_{\infty}$, we have

$$
(q-2) S(r, f) \leq \sum_{j=1}^{q} \bar{C}\left(r, \frac{1}{f-a_{j}}\right)+O(\log r T(r, f)), r \notin F,
$$

where $F$ is a set of finite linear measure. 
Lemma 3 ([19]). Suppose that $f(z)$ is a nonconstant meromorphic function and $\bar{\Omega}(\alpha, \beta)$ is an angular domain, where $0<\beta-\alpha \leq 2 \pi$. Then for any $r<R$,

$$
\begin{gathered}
A\left(r, \frac{f^{\prime}}{f}\right) \leq K\left\{\left(\frac{R}{r}\right)^{k} \int_{1}^{R} \frac{\log ^{+} T(t, f)}{t^{1+k}} d t+\log ^{+} \frac{r}{R-r}+\log \frac{R}{r}+1\right\}, \\
B\left(r, \frac{f^{\prime}}{f}\right) \leq \frac{4 k}{r^{k}} m\left(r, \frac{f^{\prime}}{f}\right) .
\end{gathered}
$$

where $k=\frac{\pi}{\beta-\alpha}$ and $K$ is a positive constant not depending on $r$ and $R$.

Lemma 4. Let $p \in \mathbb{N}$ and $p>1$ and $f(z)$ be a meromorphic function such that $\sigma_{p}(f)=\rho(0<\rho<\infty)$. A ray $L: \arg z=\theta$ is a Borel direction of iterated p-order $\rho$ of $f$ if and only if for any positive number $0<\varepsilon<\pi / 2$, the equation

$$
\limsup _{r \rightarrow \infty} \frac{\log ^{[p]} S(r, f)}{\log r}=\rho,
$$

holds in the angular domain $\Omega_{\varepsilon}:=\{z: \theta-\varepsilon \leq \arg z \leq \theta+\varepsilon\}$.

Proof. Assume that $L$ is a Borel direction of iterated $p$-order $\rho$ of $f$, and that for some $\eta\left(0<\eta<\frac{\pi}{2}\right)$ in the angular domain $\Omega_{\eta}$, we have

$$
\limsup _{r \rightarrow \infty} \frac{\log ^{[p]} S(r, f)}{\log r}<\rho .
$$

By Lemma 1 , for any finite value $a$, in the angular domain $\Omega_{\eta}$, we have $S\left(r, \frac{1}{f-a}\right)=S(r, f)+O(1)$. Since $C(r, a) \leq S\left(r, \frac{1}{f-a}\right)$, then

$$
C(r, a) \leq S\left(r, \frac{1}{f-a}\right)=S(r, f)+O(1) .
$$

On the other hand,

$$
\begin{aligned}
C(2 r, a) & \geq C_{\theta-\frac{\eta}{2}, \theta+\frac{\eta}{2}}(2 r, a) \\
& \geq 2 \sum_{1<\left|b_{v}\right|<r, \theta-\frac{\eta}{2}<\beta_{v}<\theta+\frac{\eta}{2}}\left(\frac{1}{\left|b_{v}\right|^{k}}-\frac{\left|b_{v}\right|^{k}}{(2 r)^{2 k}}\right) \sin k\left(\beta_{v}-\theta+\frac{\eta}{2}\right) \\
& \geq 2 \sum_{1<\left|b_{v}\right|<r, \theta-\frac{\eta}{3}<\beta_{v}<\theta+\frac{\eta}{3}}\left(\frac{1}{\left|b_{v}\right|^{k}}-\frac{\left|b_{v}\right|^{k}}{(2 r)^{2 k}}\right) \sin k\left(\beta_{v}-\theta+\frac{\eta}{2}\right),
\end{aligned}
$$

where $k=\frac{\pi}{\eta}$. In the sector $\triangle: 1<|b|<r, \theta-\frac{\eta}{3}<\beta<\theta+\frac{\eta}{3}$, we have $0<\frac{\eta}{6}<\beta_{v}-\theta+\frac{\eta}{2}<\frac{5 \eta}{6}<\frac{\pi}{2}$. We write a sum of above expression as a Stieltjes-integral and the partial integration of the above Stieltjes-integrals 
now results in

$$
\begin{aligned}
C(2 r, a) \geq & \int_{1}^{r} \frac{1}{t^{k}} d n(t)+\frac{1}{(2 r)^{2 k}} \int_{1}^{r} t^{k} d n(t) \\
\geq & k \int_{1}^{r} \frac{1}{t^{k+1}} n(t) d t+\frac{n(r)}{r^{k}}-\frac{r^{k} n(r)}{r^{2 k}} \\
& +\frac{k}{(2 r)^{2 k}} \int_{1}^{r} t^{k-1} n(t) d t \\
\geq & \frac{n(r)}{r^{k}}-\frac{r^{k} n(r)}{(2 r)^{2 k}} \\
\geq & \left(1-\frac{1}{2^{2 k}}\right) \frac{n(r)}{r^{k}}
\end{aligned}
$$

where a short-hand notation $n(t)=n\left(t, \Omega\left(\theta-\frac{\eta}{3}, \theta+\frac{\eta}{3}\right), f=a\right)$ will be used. Substituting (6) to (5) and combining (4), we get

$$
\limsup _{r \rightarrow \infty} \frac{\log ^{[p]} n\left(r, \Omega\left(\theta-\frac{\eta}{3}, \theta+\frac{\eta}{3}\right), f=a\right)}{\log r}<\rho .
$$

Since $a$ is arbitrary, the above expression is incompatible with the hypothesis that $L$ is a Borel direction of iterated $p$-order $\rho$ of $f$.

Conversely, assume that for any $\eta\left(0<\eta<\frac{\pi}{2}\right)$, in the angular domain $\Omega_{\eta}$, we have

$$
\limsup _{r \rightarrow \infty} \frac{\log ^{[p]} S(r, f)}{\log r}=\rho .
$$

Suppose that $L$ is not a Borel direction of iterated $p$-order $\rho$ of $f$. Then there exist a $\eta$ and three distinct values $a_{j} \in \mathbb{C}_{\infty}(j=1,2,3)$, such that for sufficiently large $r$, we have

$$
n\left(r, \Omega(\theta-\eta, \theta+\eta), f=a_{j}\right)<\exp ^{[p-1]}\left(r^{Q}\right),
$$

where $Q<\rho$ is a constant. For the three distinct value $a_{j}$, we have

$$
C\left(r, a_{j}\right) \leq 2 n\left(r, \Omega(\theta-\eta, \theta+\eta), f=a_{j}\right) .
$$

We deduce from (8), (9) and Lemma 2 that $S(r, f)<\exp ^{[p-1]}\left(r^{Q}\right)$ for sufficiently large $r$. Hence, we get a contradiction and Lemma 4 follows.

Remark 5. In the proof of Lemma 4, we noted that for some $\eta\left(0<\eta<\frac{\pi}{2}\right)$ in the angular domain $\Omega_{\eta}$, we have

$$
\limsup _{r \rightarrow \infty} \frac{\log ^{[p]} S(r, f)}{\log r} \leq \rho .
$$

In fact, if

$$
\limsup _{r \rightarrow \infty} \frac{\log ^{[p]} S(r, f)}{\log r}>\rho .
$$


By Lemma 2, we have for some $a \in \mathbb{C}_{\infty}$,

$$
\limsup _{r \rightarrow \infty} \frac{\log ^{[p]} n\left(r, \Omega_{\eta}, f=a\right)}{\log r}>\rho .
$$

This contradicts with $\sigma_{p}(f)=\rho$.

Lemma 5 ([24]). Let $f(z)$ be an analytic function on $\bar{\Omega}(\alpha, \beta)$. Then we have

$$
\log M(r, \bar{\Omega}(\alpha, \beta), f) \leq K r^{\omega}\{S(2 r, f)+1\},
$$

where $\omega=\frac{\pi}{\beta-\alpha}, M(r, \bar{\Omega}(\alpha, \beta), f)=\sup \left\{\left|f\left(t e^{i \tau}\right)\right|: \alpha \leq \tau \leq \beta, 1 \leq t \leq r\right\}$ and $K$ is a positive constant.

\section{Proof of Theorem 1}

Proof. The Wronskian determinant $W\left(f_{1}, f_{2}, \ldots, f_{k}\right)$ of fundamental system of solutions $\left\{f_{1}, f_{2}, \ldots, f_{k}\right\}$ is given by

$$
W=W\left(f_{1}, f_{2}, \ldots, f_{k}\right)=\left|\begin{array}{cccc}
1 & 1 & \cdots & 1 \\
\frac{f_{1}^{\prime}}{f_{1}} & \frac{f_{2}^{\prime}}{f_{2}} & \cdots & \frac{f_{n}^{\prime}}{f_{k}} \\
\frac{f_{1}^{(k-1)}}{f_{1}} & \frac{f_{2}^{(k-1)}}{f_{2}} & \cdots & \frac{f_{k}^{(k-1)}}{f_{k}}
\end{array}\right| .
$$

Applying a Lemma $[12$, p. 16], we can derive that $W$ is a positive constant and denote it by $K$. Hence

$$
\frac{1}{E}=\frac{1}{K} \frac{W}{E}=\frac{1}{K} \sum_{1 \leq i_{l} \neq i_{l} \leq k}(-1)^{\tau} \Pi_{l=1}^{k-1} \frac{f_{i_{l}}^{(l)}}{f_{i_{l}}} .
$$

Let $f \not \equiv 0$ be a solution of (2). It follows from Theorem $\mathrm{C}$ that the iterated $p$-order of $\log T(r, f)$ is at most $\sigma$, where $\sigma<\infty$ is a constant.

For any $\theta \in \mathbb{R}$, using Lemma 3 in which $R=2 r$ for sufficiently small $\varepsilon$, we have

$$
\begin{aligned}
A_{\theta-\varepsilon, \theta+\varepsilon}\left(r, \frac{f_{i}^{\prime}}{f_{i}}\right) & =O\left(\int_{1}^{2 r} \frac{\log ^{+} T\left(t, f_{i}\right)}{t^{1+\frac{\pi}{2 \varepsilon}}} d t\right)=O\left(\int_{1}^{2 r} \frac{\exp ^{[p-1]} t^{\sigma+1}}{t^{1+\frac{\pi}{2 \varepsilon}}} d t\right) \\
& =O\left(\exp ^{[p-1]} r^{\sigma+1}\right)
\end{aligned}
$$

for any $f_{i}$, when $p \geq 2$. And when $p=1$, we have $A_{\theta-\varepsilon, \theta+\varepsilon}\left(r, \frac{f_{i}^{\prime}}{f_{i}}\right)=O(1)$.

Since

$$
m\left(r, \frac{f_{i}^{\prime}}{f_{i}}\right)=O\left(\log r T\left(r, f_{i}\right)\right)=O\left(\exp ^{[p-1]} r^{\sigma+1}\right) .
$$

Then we can deduce the following equation from Lemma 3

$$
B_{\theta-\varepsilon, \theta+\varepsilon}\left(r, \frac{f_{i}^{\prime}}{f_{i}}\right) \leq \frac{4 k}{r^{k}} m\left(r, \frac{f_{i}^{\prime}}{f_{i}}\right)=O\left(r^{\sigma+1-\frac{\pi}{2 \varepsilon}}\right)=O\left(\exp ^{[p-1]} r^{\sigma+1}\right)
$$

when $p \geq 2$, and when $p=1$, we have $B_{\theta-\varepsilon, \theta+\varepsilon}\left(r, \frac{f_{i}^{\prime}}{f_{i}}\right)=O(1)$. 
Since

$$
D_{\theta-\varepsilon, \theta+\varepsilon}\left(r, \frac{f_{i}^{(h)}}{f_{i}}\right) \leq \sum_{i=1}^{h} D_{\theta-\varepsilon, \theta+\varepsilon}\left(r, \frac{f_{i}^{(l)}}{f_{i}^{(l-1)}}\right)+O(1),
$$

where $i=1,2, \ldots, k ; h=2,3, \ldots, k-1$, then we have

$$
D_{\theta-\varepsilon, \theta+\varepsilon}\left(r, \frac{1}{E}\right)=O(1)
$$

when $p=1$, and when $p \geq 2$,

$$
D_{\theta-\varepsilon, \theta+\varepsilon}\left(r, \frac{1}{E}\right)=O\left(\exp ^{[p-1]} r^{\sigma+1}\right) .
$$

By the definition and Lemma 1 , for any $\theta \in \mathbb{R}$ and any sufficiently small $\varepsilon>0$ in angular domain $\Omega_{\varepsilon}=\{z \mid \theta-\varepsilon \leq \arg z \leq \theta+\varepsilon\}$, we can deduce

$$
S(r, E) \leq P\left\{C\left(r, \frac{1}{E}\right)+O\left(\exp ^{[p-1]} r^{\sigma+1}\right)\right\},
$$

where $P$ is a constant.

Now, we are in the position to prove the statements those in Theorem 1 are equivalent.

\subsection{Proof of the equivalence of (i) and (ii)}

Suppose that $L: \arg z=\theta$ is a Borel direction of iterated $(p+1)$-order $\rho$ of $E$. By Lemma 4 , for any positive number $0<\varepsilon<\pi / 2$,

$$
\limsup _{r \rightarrow \infty} \frac{\log ^{[p+1]} S(r, E)}{\log r}=\rho,
$$

holds in the angular domain $\Omega_{\varepsilon}:=\{z: \theta-\varepsilon \leq \arg z \leq \theta+\varepsilon\}$. Combining (13), we can obtain

$$
\limsup _{r \rightarrow \infty} \frac{\log ^{[p+1]} C\left(r, \frac{1}{E}\right)}{\log r}=\rho .
$$

Noting $C\left(r, \frac{1}{E}\right) \leq 2 n\left(r, \Omega_{\varepsilon}, E=0\right)$, hence $\lambda_{(p+1), \theta}(E)=\rho$.

On the other hand, if $\lambda_{(p+1), \theta}(E)=\rho$, then for any $0<\eta<\frac{\pi}{2}$, in the angular domain $\Omega_{\eta}$, we can obtain the following equation as we did in the proof of (6)

$$
S(2 r, E) \geq\left(1-\frac{1}{2^{2 k}}\right) \frac{n(r)}{r^{k}}+O(1),
$$

where $n(r)=n\left(t, \Omega\left(\theta-\frac{\eta}{3}, \theta+\frac{\eta}{3}\right), E=0\right)$. Thus for any $0<\eta<\frac{\pi}{2}$,

$$
\limsup _{r \rightarrow \infty} \frac{\log ^{[p+1]} S(r, E)}{\log r}=\rho,
$$

holds in the angular domain $\Omega_{\eta}$. By Lemma 4, we know that $L: \arg z=\theta$ is a Borel direction of iterated $(p+1)$-order $\rho$ of $E$. 


\subsection{Proof of the equivalence of (i) and (iii)}

Suppose that $L: \arg z=\theta$ is a Borel direction of iterated $(p+1)$-order $\rho$ of $E$. By Lemma 4 , for any positive number $0<\mu<\pi / 2$,

$$
\limsup _{r \rightarrow \infty} \frac{\log ^{[p+1]} S(r, E)}{\log r}=\rho
$$

holds in the angular domain $\Omega_{\mu}$. If there exists $0<\varepsilon<\pi / 2$ such that

$$
\limsup _{r \rightarrow \infty} \frac{\log ^{[p+2]} M\left(r, \Omega_{\theta, \varepsilon}, E\right)}{\log r}<T<\rho .
$$

Then we have

$$
\log \left|E r e^{i(\theta-\varepsilon)}\right|<\exp ^{[p]} r^{T}, \quad \log \left|E r e^{i(\theta+\varepsilon)}\right|<\exp ^{[p]} r^{T}
$$

and $\log \left|E r e^{i \eta}\right|<\exp ^{[p]} r^{T}$ for any large $r$ and all $\theta \in[\theta-\varepsilon, \theta+\varepsilon]$. Noting that $E$ is an entire function, we deduce from the definition of the Nevanlinna angular characteristic that

$$
\limsup _{r \rightarrow \infty} \frac{\log ^{[p+1]} S(r, E)}{\log r}<\rho .
$$

This contradicts with (16). Hence, for any $0<\varepsilon<\pi / 2$, we have

$$
\limsup _{r \rightarrow \infty} \frac{\log ^{[p+2]} M\left(r, \Omega_{\theta, \varepsilon}, E\right)}{\log r}=\rho .
$$

On the other hand, if

$$
\limsup _{r \rightarrow \infty} \frac{\log ^{[p+2]} M\left(r, \Omega_{\theta, \varepsilon}, E\right)}{\log r}=\rho
$$

for any $0<\varepsilon<\pi / 2$, by using Lemma 5 and Remark 5 , we have

$$
\limsup _{r \rightarrow \infty} \frac{\log ^{[p+1]} S(r, E)}{\log r}=\rho
$$

holds in the angular domain $\Omega_{\varepsilon}$. It follows Lemma 4 that $L: \arg z=\theta$ is a Borel direction of iterated $(p+1)$-order $\rho$ of $E$.

Acknowledgments. The author would like to thank referees for providing numerous suggestions and comments. This research was partially supported by the NSF of Jiangxi Province (Grant 2010GZC0187) and by NSF of Educational Department of the Hubei Province (Grant T201009, Q20112807).

\section{References}

[1] S. B. Bank and I. Laine, On the oscillation theory of $f^{\prime \prime}+A f=0$ where $A$ is entire, Trans. Amer. Math. Soc. 273 (1982), no. 1, 351-363.

[2] S. B. Bank and J. K. Langley, Oscillation theory for higher order linear differential equations with entire coefficients, Complex Variables Theory Appl. 16 (1991), no. 2-3, $163-175$. 
[3] L. G. Bernal, On growth k-order of solutions of a complex homogeneous linear differential equation, Proc. Amer. Math. Soc. 101 (1987), no. 2, 317-322.

[4] T.-B. Cao, Complex oscillation of entire solutions of higher-order linear differential equations, Electron. J. Differential Equations 2006 (2006), no. 81, 8 pp. (electronic).

[5] C. Chuang, Differential Polynomials of Meromorphic Functions, Beijing Normal University Press, Beijing, 1999.

[6] S. Gao, Z. Chen, and T. Chen, Oscillation Theory of Linear Differential Equation, Huazhong University of Science and Technology Press, Wuhan, 1998.

[7] Z. Huang and Z. Chen, Angular distribution with hyper-order in complex oscillation theory, Acta Math. Sinica (Chin. Ser.) 50 (2007), no. 3, 601-614.

[8] angular domain, Bull. Korean Math. Soc. 47 (2010), no. 3, 443-454.

[9] - The zeros distribution of hyper solutions of higher order differential equations in angular domain, Southeast Asian Bull. Math. 34 (2010), no. 3, 429-438.

[10] G. Jank and L. Volkmann, Meromorphe funktionen und Differentialiagleichungen, Birkhäuser, 1985.

[11] L. Kinnunen, Linear differential equations with solutions of finite iterated order, Southeast Asian Bull. Math. 22 (1998), no. 4, 385-405.

[12] I. Laine, Nevanlinna theory and complex differential equations, de Gruyter Studies in Mathematics, 15. Walter de Gruyter Co., Berlin, 1993.

[13] R. Nevanlinna, über eine Klasse meromorpher Funktionen, Math. Ann. 92 (1924), no. $3-4,145-154$.

[14] J. Rossi and S. Wang, The radial oscillation of solutions to ODE's in the complex domain, Proc. Edinburgh Math. Soc. (2) 39 (1996), no. 3, 473-483.

[15] D. Sato, On the rate of growth of entire functions of fast growth, Bull. Amer. Math. Soc. 69 (1963), 411-414.

[16] M. Tsuji, Potential Theory in Modern Function Theory, Maruzen Co. Ltd, Tokyo, 1959.

[17] S. Wang, On the sectorial oscillation theory of $f^{\prime \prime}+A(z) f=0$, Ann. Acad. Sci. Fenn. Ser. A I Math. Dissertationes (1994), no. 92, 66 pp.

[18] S. Wu, Angular distribution in complex oscillation, Sci. China Ser. A 48 (2005), no. 1, $107-114$.

[19] - On the location of zeros of solution of $f^{\prime \prime}+A f=0$ where $A(z)$ is entire, Math. Scand. 74 (1994), no. 2, 293-312.

[20] Z. Wu and D. Sun, Angular distribution of solutions of higher order linear differential equations, J. Korean Math. Soc. 44 (2007), no. 6, 1329-1338.

[21] Sectorial oscillation of linear differential equations and iterated order, Electron. J. Differential Equations 2007 (2007), no. 134, 7 pp. (electronic).

[22] L. Yang, Value Distribution Theory, Translated and revised from the 1982 Chinese original. Springer-Verlag, Berlin; Science Press Beijing, Beijing, 1993.

[23] C. Yi, The augular distribution of the solutions of higher order differential equation, Acta Math. Sinica (Chin. Ser.) 48 (2005), no. 1, 133-140.

[24] J. Zheng, Value Distrbution of Meromorphic Functions, Springer, Beijing: Tsinghua Univ. Press. 2010

SCHOOL OF MATHEMATICS AND STATISTICS

Hubei University of Science and Technology

Xianning, Hubei Province, 437100, P. R. China

E-mail address: wuzj52@hotmail.com 